\title{
SUBARCSECOND MID-INFRARED OBSERVATIONS OF NGC 6240: LIMITATIONS OF ACTIVE GALACTIC NUCLEUS-STARBURST POWER DIAGNOSTICS ${ }^{1}$
}

\author{
E. Egami, ${ }^{2,3}$ G. Neugebauer, ${ }^{2,3}$ B. T. Soifer, ${ }^{2,4}$ K. Matthews, ${ }^{2}$ E. E. Becklin, ${ }^{5}$ And M. E. Ressler ${ }^{6}$ \\ Received 2005 June 23; accepted 2005 November 3
}

\begin{abstract}
In order to examine the relative importance of powerful starbursts and Compton-thick active galactic nuclei (AGNs) in NGC 6240, we have obtained mid-infrared images and low-resolution spectra of the galaxy with subarcsecond spatial resolution using the Keck telescopes. Despite the high spatial resolution $(\sim 200 \mathrm{pc})$ of our data, no signature of the hidden AGNs has been detected in the mid-infrared. The southern nucleus, which we show provides $80 \%-90 \%$ of the total $8-25 \mu \mathrm{m}$ luminosity of the system, has a mid-infrared spectrum and a mid-/far-infrared spectral energy distribution consistent with starbursts. At the same time, however, it is also possible to attribute up to $60 \%$ of the bolometric luminosity to an AGN, consistent with X-ray observations, if the AGN is heavily obscured and emits mostly in the far-infrared. This ambiguity arises because the intrinsic variation of properties among a given galaxy population (e.g., starbursts) introduces at least a factor of a few uncertainty even into the most robust AGN-starburst diagnostics. We conclude that with present observations it is not possible to determine the dominant power source in galaxies when AGN and starburst luminosities are within a factor of a few of each other.
\end{abstract}

Key words: galaxies: active — galaxies: individual (NGC 6240) — galaxies: interactions — galaxies: nuclei — galaxies: starburst — infrared: galaxies

\section{INTRODUCTION}

NGC 6240 is a nearby $(D \sim 100 \mathrm{Mpc})^{7}$ system of interacting galaxies that epitomizes the active galactic nucleus (AGN)starburst controversy surrounding ultraluminous infrared galaxies (ULIRGs). ${ }^{8}$ Together with Arp 220 (Soifer et al. 1984), NGC 6240 was one of the first few galaxies discovered by IRAS to have an extremely large $\left(>10^{11}-10^{12} L_{\odot}\right)$ and dominant $\left(>0.9 L_{\text {bol }}\right)$ infrared luminosity (Wright et al. 1984). Its highly disturbed morphology (Fosbury \& Wall 1979) and two nuclei separated by less than $2^{\prime \prime}(<1 \mathrm{kpc}$; Fried \& Schulz 1983) indicate that this is a system of two merging galaxies. Based on the shape of the infrared spectral energy distribution (SED) and the large infrared size $(>3 \mathrm{kpc})$ inferred initially, it was argued that powerful starbursts induced by galaxy interaction generate the large infrared luminosity by heating interstellar dust with the UV

\footnotetext{
${ }^{1}$ The data presented herein were obtained at the W. M. Keck Observatory, which is operated as a scientific partnership among the California Institute of Technology, the University of California, and the National Aeronautics and Space Administration. The Observatory was made possible by the generous financial support of the W. M. Keck Foundation.

2 Caltech Optical Observatories, California Institute of Technology, Mail Code 105-24, Pasadena, CA 91125.

${ }^{3}$ Current address: Steward Observatory, University of Arizona, 933 North Cherry Avenue, Tucson, AZ 85721.

${ }^{4}$ Spitzer Science Center, California Institute of Technology, Mail Code 314-6, Pasadena, CA 91125

5 Department of Physics and Astronomy, University of California, Los Angeles, CA 90095-1562.

${ }^{6}$ Jet Propulsion Laboratory, California Institute of Technology, 4800 Oak Grove Drive, Pasadena, CA 91109.

${ }^{7}$ We adopt a distance of $103.86 \mathrm{Mpc}$ derived by Sanders et al. (2003) based on a redshift of 0.0243 (Solomon et al. 1997) and the cosmic attractor model of Mould et al. (2000). The assumed cosmological parameters are $\Omega_{M}=0.3$, $\Omega_{\Lambda}=0.7$, and $H_{0}=75 \mathrm{~km} \mathrm{~s}^{-1} \mathrm{Mpc}^{-1}$, respectively. At this distance and redshift, $1^{\prime \prime}$ subtends 490 pc.

${ }^{8}$ With an infrared luminosity $L_{\mathrm{IR}}=L(8-1000 \mu \mathrm{m})$ as defined by Sanders \& Mirabel (1996) of $7.1 \times 10^{11} L_{\odot}$ (Sanders et al. 2003), NGC 6240 is a luminous infrared galaxy $\left(\mathrm{LIRG} ; L_{\mathrm{IR}}>10^{11} L_{\odot}\right)$ rather than a ULIRG $\left(L_{\mathrm{IR}}>10^{12} L_{\odot}\right)$ in the strict sense. However, we do not make this distinction here, because NGC 6240 shares many of the ULIRG properties.
}

radiation from young massive stars (Wright et al. 1984; Joseph \& Wright 1985).

Although the size argument later turned out to be incorrect when the mid-infrared size was measured to be $<500 \mathrm{pc}$ by Wynn-Williams \& Becklin (1993), this starburst hypothesis for NGC 6240 has subsequently gained wide support from a series of follow-up observations. The most crucial pieces of evidence are (1) the deep CO absorption band heads, (2) the strong polycyclic aromatic hydrocarbon (PAH) features, and (3) the starburstlike radio-infrared luminosity ratio. The deep CO absorption band heads detected in the near-infrared clearly indicate that the nearinfrared continuum of NGC 6240 is dominated by starlight (Rieke et al. 1985; Lester et al. 1988; Ridgway et al. 1994; Shier et al. 1996; Sugai et al. 1997; Tecza et al. 2000). The most recent study by Tecza et al. (2000) shows that these CO absorption band heads are likely to be produced by late-K or early-M supergiants, and that the starburst population associated with these supergiants, estimated to be triggered $\sim 2 \times 10^{7} \mathrm{yr}$ ago and lasting $\sim 5 \times 10^{6} \mathrm{yr}$, is powerful enough to provide $30 \%-100 \%$ of the bolometric luminosity. The strong PAH features seen in the mid-infrared spectra of NGC 6240 (Smith et al. 1989; Genzel et al. 1998; Dudley 1999; Rigopoulou et al. 1999; Imanishi \& Dudley 2000; Lutz et al. 2003) also suggest starbursts as the main luminosity source, since they are much weaker in AGN galaxies. Finally, the radioinfrared luminosity ratio of NGC 6240, averaged over the whole galaxy, is also starburst-like (Colbert et al. 1994; Tecza et al. $2000)$, although the existence of compact radio cores $(<26 \mathrm{pc})$ in the two nuclei may suggest some AGN radio emission (Colbert et al. 1994; Beswick et al. 2001; Gallimore \& Beswick 2004). Together with the starburst-driven superwind seen both in the visual (Heckman et al. 1987, 1990; Armus et al. 1990) and in the soft X-ray (Schulz et al. 1998; Komossa et al. 1998; Iwasawa \& Comastri 1998; Lira et al. 2002), the evidence for the powerful starbursts is indeed strong.

This starburst picture was seriously challenged by a series of strong hard X-ray detections, which suggest the existence of a QSO-like AGN in NGC 6240. An initial hint came from the $A S C A$ 
hard X-ray spectrum, which showed an extremely flat continuum above $3 \mathrm{keV}$ with a strong iron K line complex (Mitsuda 1995; Kii et al. 1997; Iwasawa \& Comastri 1998; Nakagawa et al. 1999). This suggests that what we are seeing is reflected X-ray emission from an AGN obscured by Compton-thick $\left(N_{\mathrm{H}}>2 \times 10^{24} \mathrm{~cm}^{-2}\right)$ material, and that the intrinsic luminosity of this AGN could be as high as $2.6 \times 10^{11} L_{\odot}\left(10^{45} \mathrm{ergs} \mathrm{s}^{-1}\right.$; Iwasawa \& Comastri 1998). This conclusion was later confirmed by the BeppoSAX observations, which showed that the X-ray spectrum of NGC 6240 clearly exhibits the emergence of a powerful hard X-ray continuum above $10 \mathrm{keV}$ from the obscured AGN (Vignati et al. 1999). If we take the estimated intrinsic $2-10 \mathrm{keV}$ nuclear luminosity of $>10^{44} \mathrm{ergs} \mathrm{s}^{-1}$ (absorption-corrected based on the BeppoSAX spectrum extending up to $100 \mathrm{keV}$ ) and assume that it is less than $10 \%$ of the AGN bolometric luminosity $\left(L_{2}-10 \mathrm{keV} / L_{\mathrm{bol}} \sim\right.$ 0.03-0.1 for Seyfert galaxies and QSOs, according to Iwasawa et al. [2001]), this sets a lower limit of $10^{45} \mathrm{ergs} \mathrm{s}^{-1}$ on the AGN luminosity in NGC 6240, which is consistent with the ASCA result. Given various uncertainties, the $\mathrm{AGN}$ can also produce $50 \%-100 \%$ of the bolometric luminosity of NGC 6240 . Recently, Chandra hard X-ray images of NGC 6240 have shown that this hard X-ray emission originates from two nuclei (Komossa et al. 2003). A detailed X-ray spectroscopic study has also been performed by Boller et al. (2003) and Netzer et al. (2005) based on the XMM-Newton X-ray data.

In the last few years, NGC 6240 has been studied with increasingly high spatial resolution. Gerssen et al. (2004) resolved both nuclei into separate components using Hubble Space Telescope (HST) observations at visual wavelengths, but they found no clear sign of the two AGNs. They emphasize, however, the importance of X-ray data for identifying AGNs in highly dustenshrouded environments. Very Long Baseline Array (VLBA) observations at radio frequencies reveal three compact sources in NGC 6240 (Gallimore \& Beswick 2004), two of which are associated with the X-ray nuclei.

The two conflicting pictures put NGC 6240 at the heart of the AGN-starburst controversy within ULIRGs: that is, do AGNs or starbursts dominate their luminosity output? In recent years, the prevailing view has been that it is starbursts that dominate. The majority of ULIRGs show no obvious sign of a hidden AGN, and the observed starburst components seem powerful enough to provide the bolometric luminosity of these galaxies when corrected for extinction (Genzel et al. 1998). However, NGC 6240 is a notable anomaly in this picture, with strong evidence for a dust-obscured, powerful AGN. If this AGN indeed provides a substantial fraction of the infrared luminosity, the validity of various AGN-starburst power diagnostics must be reexamined, since many of these diagnostics indicate NGC 6240 to be a typical starburst galaxy. Most recently, the existence of a Compton-thick AGN was also hinted for Arp 220, another ULIRG with no sign of an AGN signature in the infrared, through the detection of the Fe $\mathrm{K}$ emission in the hard X-ray (Iwasawa et al. 2005). The SED modeling of Arp 220 by Spoon et al. (2004) suggests that such a heavily obscured AGN might contribute significantly to the bolometric luminosity.

Here we present mid-infrared images and spectra of NGC 6240 obtained with the Keck telescopes with subarcsecond spatial resolutions. The Keck telescopes routinely deliver a diffractionlimited spatial resolution of $0.3-0.5$ in the mid-infrared, which corresponds to $150-250 \mathrm{pc}$ at the distance of NGC 6240. Such a high spatial resolution is essential to probe a complicated merging system like NGC 6240, in which the two nuclei are separated by only 1".4-1."5 (1".5 in VLBA [Gallimore \& Beswick 2004]; 1".4 in X-rays [Komossa et al. 2003]).

\section{OBSERVATIONS AND DATA REDUCTION}

\subsection{Keck II MIRLIN Mid-Infrared Images}

The mid-infrared images of NGC 6240 were taken on UT 1998 March 19 with the MIRLIN camera (Ressler et al. 1994) on the Keck II 10 m telescope on Mauna Kea in Hawaii. The camera used a $128 \times 128 \mathrm{Si}:$ As BIB array and was attached to the $\mathrm{f} / 40$ bent Cassegrain visitor port, producing a pixel scale of 0 . 138 pixel $^{-1}$ with a field of view $18^{\prime \prime}$ on a side. Secondarymirror chopping and telescope nodding were employed for effective subtraction of the sky background and instrumental noise. The observational methods and characteristics, as well as the photometric calibration and data reduction, were the same as those used by Soifer et al. $(1999,2000)$ and are described therein.

Observations of NGC 6240 were made in the seven midinfrared filters listed in Soifer et al. (1999), where the central wavelengths and filter full widths are given. The central wavelengths are also listed in Table 1, together with the on-source integration times. The mean air mass was 1.07. The MIRLIN observations emphasized photometry rather than spatial structure, and as a result no strict size limit can be placed on the observations of the nucleus of NGC 6240. In particular, since no star was observed close in time, we do not have accurate estimates of the point-spread function and seeing. However, the bright compact southern nucleus of NGC 6240 (Fig. 1) has FWHMs of 0."50 ".7 at $7.9-24.5 \mu \mathrm{m}$, indicating that the intrinsic seeing was at least this good.

\subsection{Keck I LWS Mid-Infrared Spectra}

The low-resolution $(\Delta \lambda / \lambda \sim 50)$ mid-infrared spectra of NGC 6240 were obtained with the Long Wavelength Spectrometer (LWS; Jones \& Puetter 1993) on the Keck I 10 m telescope. The LWS uses a $128 \times 128 \mathrm{BIB}$ array and is attached to the $\mathrm{f} / 25$ forward Cassegrain focus of the telescope, producing a pixel scale of 0.08 pixel $^{-1}$ with a field of view $10^{\prime \prime}$ on a side. The slit width was 0 ".48 (6 pixels). The observing methods, instrumental characteristics, and data reduction used to obtain the mid-infrared spectra are the same as those used by Soifer et al. (2002) and are described therein.

The spectra of NGC 6240 covering $8.8-13.1 \mu \mathrm{m}$ were taken on UT 2000 May 21, while those covering 7.5-12.1 $\mu \mathrm{m}$ were taken on UT 2000 May 22. The total integration times are 147 and 59 minutes, respectively. The slit position angle was $16^{\circ}$ (east of north), which is along the direction connecting the two nuclei. The NGC 6240 spectra were divided by the spectra of HR 5340 and HR 6406 and multiplied by a blackbody spectrum at the star's effective temperature. For wavelength calibration, we adopted the spectral dispersion of $0.0375 \mu \mathrm{m} \mathrm{pixel}^{-1}$ derived from our other LWS data and adjusted the offset by using the PAH $11.3 \mu \mathrm{m}$ feature seen redshifted to $11.6 \mu \mathrm{m}$ in the NGC 6240 spectra.

\section{RESULTS}

\subsection{Mid-Infrared Morphology and Photometry}

Figure 1 shows the mid-infrared images. The images clearly show two compact nuclei separated by $\sim 1$ ". $6 \pm 0$ ". 1 along the position angle of $16^{\circ}$ (east of north). The southern nucleus dominates the mid-infrared luminosity output. It appears round and symmetric, although some north-south elongation is seen in the 11.7 and $12.5 \mu \mathrm{m}$ images, which are of the highest signal-tonoise ratio. The subcomponents of the two main sources seen by Max et al. (2005) in the near-infrared and by Gallimore \& 
TABLE 1

NGC 6240 Mid-Infrared Continuum Flux Densities

\begin{tabular}{|c|c|c|c|c|}
\hline $\begin{array}{c}\text { Wavelength } \\
\qquad(\mu \mathrm{m})\end{array}$ & $\begin{array}{l}T_{\text {int }} \\
(\mathrm{s})\end{array}$ & $\begin{array}{l}\text { Total }^{\mathrm{a}} \\
(\mathrm{mJy})\end{array}$ & $\begin{array}{l}\text { South }^{\mathrm{b}} \\
\text { (mJy) }\end{array}$ & $\begin{array}{l}\text { North }^{\mathrm{b}} \\
\text { (mJy) }\end{array}$ \\
\hline $7.9 \ldots$ & 360 & $494 \pm 12$ & $275 \pm 3$ & $59 \pm 3$ \\
\hline $8.8 \ldots \ldots$ & 360 & $243 \pm 6$ & $142 \pm 1$ & $29 \pm 1$ \\
\hline $10.3 \ldots \ldots \ldots \ldots \ldots \ldots \ldots \ldots$ & 315 & $110 \pm 8$ & $67 \pm 2$ & $7 \pm 2$ \\
\hline $11.7 \ldots$ & 360 & $316 \pm 4$ & $201 \pm 1$ & $41 \pm 1$ \\
\hline $12.5 \ldots \ldots$ & 360 & $480 \pm 6$ & $375 \pm 2$ & $58 \pm 2$ \\
\hline 17.9 & 480 & $1098 \pm 30$ & $919 \pm 9$ & $119 \pm 9$ \\
\hline $24.5 .$. & 432 & $3263 \pm 112$ & $2734 \pm 31$ & $384 \pm 31$ \\
\hline
\end{tabular}

Notes.-The uncertainties listed in the table are statistical only. Photometric uncertainties in the MIRLIN data are $\pm 5 \%$ for $<20 \mu \mathrm{m}$ and $\pm 10 \%$ for $>20 \mu \mathrm{m}$.

a Measured with a 4 " diameter circular beam centered between the two nuclei.

${ }^{\mathrm{b}}$ Measured with a $1^{\prime \prime}$ diameter circular beam centered on the nucleus.
Beswick (2004) with the VLBA cannot be accurately resolved with the present mid-infrared resolution, but the elongations in the mid-infrared images hint at this structure.

Ignoring the elongation, we have estimated the source size of the southern nucleus by fitting a circularly symmetric Gaussian to the observations. Since we do not have the data to determine the seeing at the time of the NGC 6240 observations, we simply subtracted in quadrature the diffraction-limited beam sizes from the measured Gaussian FWHMs and derived the most indicative upper limits on the intrinsic size. There was, however, no evidence that its size at any of the mid-infrared wavelengths exceeded $200 \mathrm{pc}(0.4)$ after diffraction broadening of the images was accounted for.

Table 1 lists the continuum flux densities of the two nuclei measured in a circular $1^{\prime \prime}$ diameter beam, as well as the total flux densities for the whole system measured in a $4^{\prime \prime}$ diameter circular beam. The total flux densities at 12.5 and $24.5 \mu$ m measured by MIRLIN are 0.5 and $3.3 \mathrm{Jy}$, respectively, which are close to the IRAS flux densities of 0.6 and $3.4 \mathrm{Jy}$ at 12 and $25 \mu \mathrm{m}$. This indicates that these two compact nuclei provide almost all the mid-infrared luminosity of NGC 6240 . The southern nucleus is

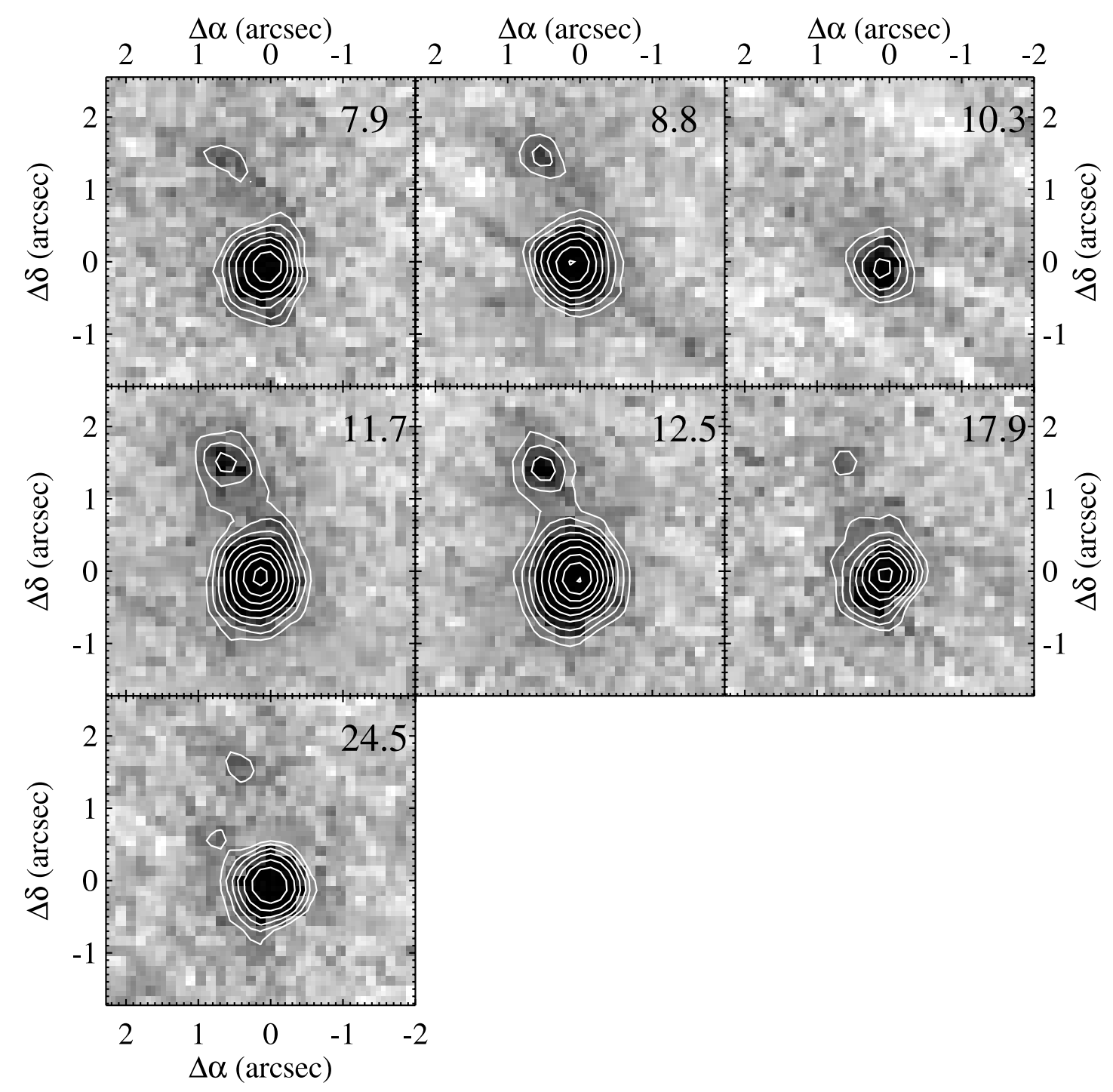

FIG. 1.-Mid-infrared images of NGC 6240. North is up and east is left. The observed wavelength (in microns) is denoted in each panel. The contour maps were produced from Gaussian-smoothed images (Gaussian FWHM $=2$ pixels). The lowest contour corresponds to $2 \sigma$ above the sky level, which was calculated in the nonsmoothed image, and the successive contours correspond to factor of 1.4 increases in surface brightness. The diagonal lines running from the upper left to lower right seen in some images (e.g., at $8.8 \mu \mathrm{m}$ ) are due to the noise pattern of the array. 
TABLE 2

NGC 6240 Continuum Flux Ratios

\begin{tabular}{|c|c|c|}
\hline Band & Flux Ratio & References \\
\hline $0.2-10 \mathrm{keV}$ & $2.8^{\mathrm{a}}$ & 1 \\
\hline $1.1 \mu \mathrm{m}$ & 2.7 & 2 \\
\hline $1.6 \mu \mathrm{m}$ & 3.0 & 2 \\
\hline $2.2 \mu \mathrm{m}$ & 3.5 & 2 \\
\hline $7.9 \mu \mathrm{m}$ & $4.7 \pm 0.2$ & 3 \\
\hline $8.8 \mu \mathrm{m}$ & $4.9 \pm 0.2$ & 3 \\
\hline $10.3 \mu \mathrm{m}$ & $>9.6^{\mathrm{b}}$ & 3 \\
\hline $11.7 \mu \mathrm{m}$ & $4.9 \pm 0.1$ & 3 \\
\hline $12.5 \mu \mathrm{m}$ & $6.5 \pm 0.2$ & 3 \\
\hline $17.9 \mu \mathrm{m}$ & $7.7 \pm 0.6$ & 3 \\
\hline $24.5 \mu \mathrm{m}$ & $7.1 \pm 0.6$ & 3 \\
\hline $1.3 \mathrm{~mm}$ & $3.2 \pm 0.3$ & 4 \\
\hline $2 \mathrm{~cm}$ & $2.5 \pm 0.5$ & 5 \\
\hline $3.6 \mathrm{~cm}$ & $2.2 \pm 0.2$ & 6 \\
\hline $6 \mathrm{~cm}$ & 2.3 & 7 \\
\hline $20 \mathrm{~cm}$ & 2.1 & 7 \\
\hline
\end{tabular}

${ }^{\mathrm{a}}$ Corrected for absorption.

${ }^{\mathrm{b}}$ We treat the $3.5 \sigma$ detection of the northern nucleus at $10.3 \mu \mathrm{m}$ (Table 1) as an upper limit.

References.- (1) Komossa et al. 2003; (2) Scoville et al. 2000;

(3) this work; (4) Tacconi et al. 1999; (5) Carral et al. 1990;

(6) Colbert et al. 1994; (7) Beswick et al. 2001.

especially luminous, providing $\sim 80 \%-90 \%$ of the total flux at these wavelengths.

Table 2 compares the flux ratio of the two nuclei at a number of wavelengths from X-ray to radio. The flux ratio increases by a factor of 2 from near-infrared to mid-infrared, indicating that the southern nucleus is slightly redder than the northern one in this wavelength range. However, the ratios are quite similar in the X-ray and radio $(\sim 2-3)$.

Figure 2 compares the MIRLIN $12.5 \mu \mathrm{m}$ image, which has the highest signal-to-noise ratio, with the HST NICMOS 1.1 and $2.2 \mu \mathrm{m}$ images (Scoville et al. 2000). The positions of the two radio nuclei (Gallimore \& Beswick 2004) are also overlaid (crosses), with the assumption that the positions of the southern nucleus are spatially coincident. Note that it is not possible to perform independent astrometric calibration with the midinfrared images because NGC 6240 is the only detected source in the small field of view of the MIRLIN images. As the figure shows, the separation and position angle of the two nuclei at $12.5 \mu \mathrm{m}$ are almost exactly the same as those at radio, and hence $\mathrm{X}$-ray, wavelengths. This suggests that we are looking at the same components in the mid-infrared as seen at the other wavelengths.

The HST NICMOS images, on the other hand, show a slightly larger separation between the nuclei, and the northern nucleus is significantly displaced from the radio position. In fact, Gerssen et al. (2004) have identified a secondary peak in the HST NICMOS images of the northern nucleus of NGC 6240 close to the position of the radio source. Its faintness in the $1.1 \mu$ m image (Fig. $2 f$ ) indicates that this source is likely to be heavily extincted by dust absorption in the galaxy. The positional coincidence argues that this fainter secondary component is responsible for the mid-infrared and radio luminosity of the northern nucleus. The southern nucleus also displays a substantial north-south elongation in the $H S T$ NICMOS images due to another secondary component seen conspicuously in the $1.1 \mu \mathrm{m}$ image (Fig. $2 f$ ).

\subsection{Mid-Infrared Spectra}

Figure $3 a$ shows the LWS two-dimensional spectrum (wavelength vs. location along the slit) of NGC 6240 covering the
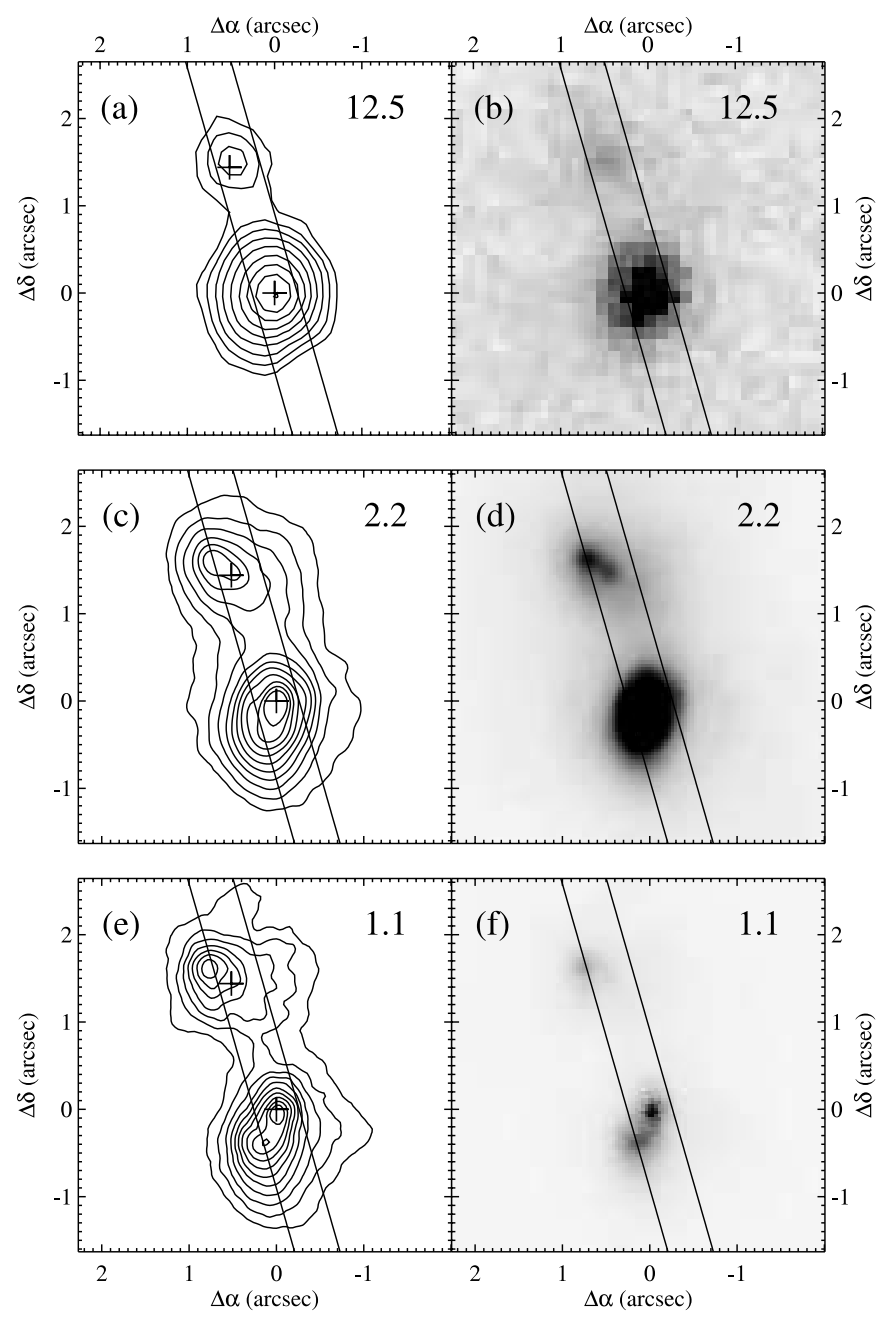

Fig. 2.-The $12.5 \mu \mathrm{m}$ image (panels $a$ and $b$ ) compared with the HST NICMOS images at $2.2 \mu \mathrm{m}$ (panels $c$ and $d$ ) and $1.1 \mu \mathrm{m}$ (panels $e$ and $f$ ) from Scoville et al. (2000). North is up and east is left. The left panels show the contour map, while the right panels show the image itself with different gray scales to emphasize the two sources in each nucleus. The contour maps were produced from Gaussian-smoothed images (Gaussian FWHM $=2$ pixels). The lowest contour corresponds to $2 \sigma$ above the sky level, which was calculated in the nonsmoothed image, and the successive contours correspond to factor of 1.4 increases in surface brightness. The two crosses overlaid on the contour plots indicate the positions of the two radio peaks (Gallimore \& Beswick 2004), with the assumption that the southern nucleus is spatially coincident at radio and mid-infrared wavelengths. The slit position of the LWS spectra, which has a position angle of $16^{\circ}$ (east of north), is also overlaid.

wavelength range from 9.9 to $12.4 \mu \mathrm{m}$ in the rest frame. The two spectra have been combined to form this two-dimensional spectrum. The actual slit position is shown in Figure 2. The southern nucleus is clearly visible, but the northern nucleus, at the position of the upper dashed line, is too faint to see. We therefore exclude the northern nucleus from the following discussion. The southern nucleus has a detectable but faint continuum at $\lesssim 11 \mu \mathrm{m}$, which rises sharply toward longer wavelengths. The prominent bright spot between 11 and $11.5 \mu \mathrm{m}$ is due to the $11.3 \mu \mathrm{m}$ PAH feature.

Figure $3 b$ shows the same spectral image with the continuum subtracted from the southern nucleus. The continuum subtraction was done in the manner described in Soifer et al. (2002). The $11.3 \mu \mathrm{m}$ PAH feature is clearly seen. However, the lineemitting region seems to be displaced from the continuum by 0.2 ( $\sim 90 \mathrm{pc})$ downward, which corresponds to the southwest 

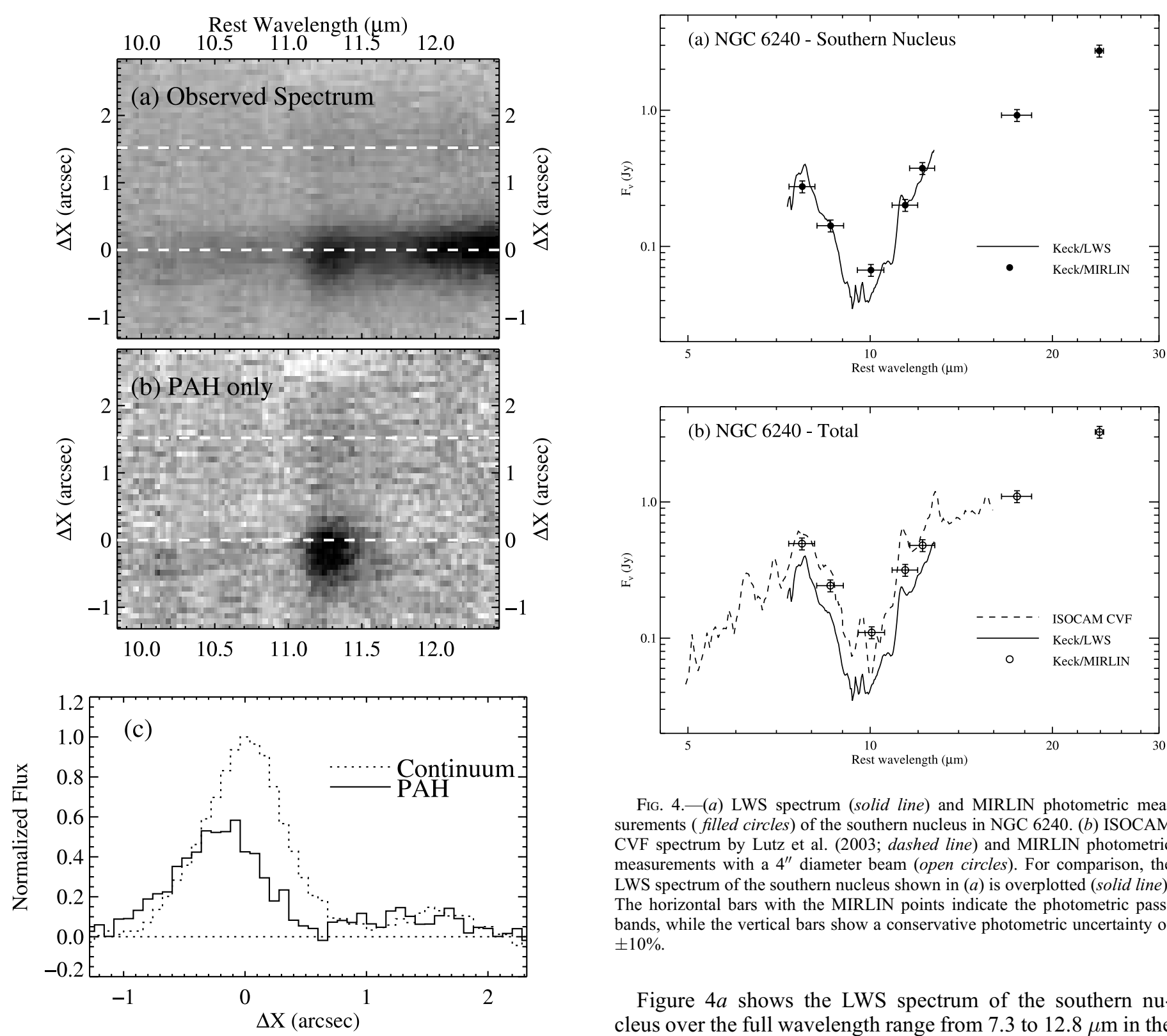

FIG. 3.-Two-dimensional LWS spectrum of NGC 6240 covering 9.9-12.4 $\mu \mathrm{m}$ in the rest frame. (a) The observed spectrum; $(b)$ the continuum-subtracted spectrum, showing a strong $11.3 \mu \mathrm{m}$ PAH feature in the southern nucleus; $(c)$ the spatial profiles of the continuum (dotted line) and $11.3 \mu \mathrm{m}$ PAH feature (solid line) produced by summing the $11.0-11.7 \mu \mathrm{m}$ region. Normalization of both components was achieved by making the integration of the continuum peak of the southern nucleus equal to unity. The dashed lines in $(a)$ and $(b)$ indicate the positions of the northern (upper lines) and southern (lower lines) nucleus, although the northern nucleus is too faint to see.

direction. This can be clearly seen when the spatial profiles of the continuum and PAH feature are compared (Fig. 3c). Incidentally, this separation is similar to that of the two near-infrared peaks in the southern nucleus seen in the HST NICMOS $1.1 \mu \mathrm{m}$ image (Figs. $2 e$ and $2 f$ ), suggesting a possible connection between the PAH peak and the secondary near-infrared peak. We also note that there is no obvious detection of the PAH emission from the molecular gas concentration between the two nuclei detected with the near-infrared $\mathrm{H}_{2}$ emission (Herbst et al. 1990; van der Werf et al. 1993; Sugai et al. 1997; Tecza et al. 2000; Bogdanović et al. 2003) and with the millimeter CO emission (Tacconi et al. 1999).

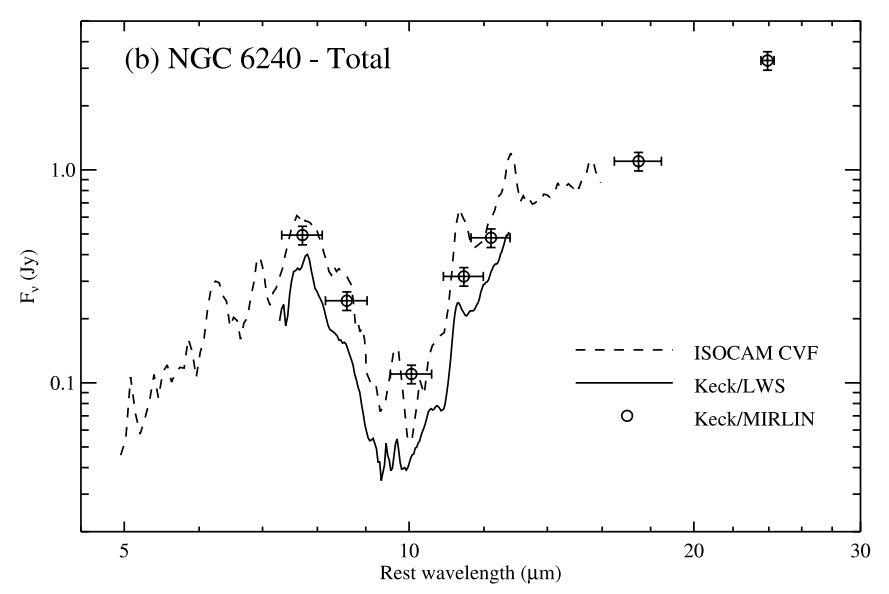

FIG. 4.-(a) LWS spectrum (solid line) and MIRLIN photometric measurements ( filled circles) of the southern nucleus in NGC 6240. (b) ISOCAM CVF spectrum by Lutz et al. (2003; dashed line) and MIRLIN photometric measurements with a $4^{\prime \prime}$ diameter beam (open circles). For comparison, the LWS spectrum of the southern nucleus shown in $(a)$ is overplotted (solid line). The horizontal bars with the MIRLIN points indicate the photometric passbands, while the vertical bars show a conservative photometric uncertainty of $\pm 10 \%$.

Figure $4 a$ shows the LWS spectrum of the southern nucleus over the full wavelength range from 7.3 to $12.8 \mu \mathrm{m}$ in the rest frame, together with the photometric measurements with MIRLIN. To produce the one-dimensional spectrum, the signals within 7 pixels $(0.56)$ of the continuum center were coadded. This extraction aperture is large enough to contain most of the continuum and PAH emission, and therefore the 0.2 offset of the PAH-emitting region mentioned above does not affect the resulting spectrum significantly (the effect on the PAH flux is an $\sim 5 \%$ reduction at most). The flux level of the spectrum was adjusted such that it makes a reasonable match to the broadband MIRLIN photometric points. A strong PAH $11.3 \mu \mathrm{m}$ feature is seen, and its line flux is measured to be $8.6 \times 10^{-16} \mathrm{~W} \mathrm{~m}^{-2}$. Also, the sharp drop of flux at the short-wavelength end of the spectrum suggests that we are seeing the peak of the $7.7 \mu \mathrm{m} \mathrm{PAH}$ feature, while the shoulder to the right indicates the existence of the $8.6 \mu \mathrm{m}$ PAH feature.

For comparison, the ISOCAM circular variable filter (CVF) spectrum presented by Lutz et al. (2003) is shown in Figure $4 b$. (Lutz et al. [2003] also give ISO Short Wavelength Spectrometer [SWS] data, but these are high-resolution spectra targeted on individual lines.) Since the ISOCAM CVF spectrum contains the fluxes from both nuclei, it is compared with the total flux measurements by MIRLIN with a $4^{\prime \prime}$ diameter circular 


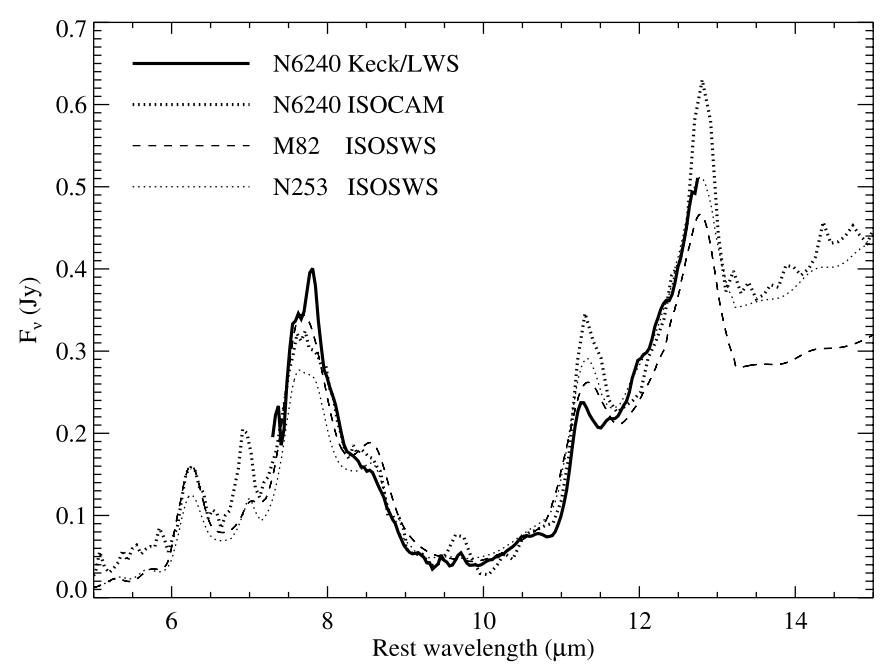

Fig. 5.-Mid-infrared spectra of the southern nucleus of NGC 6240 compared with those of the local starburst galaxies M82 and NGC 253. The thick solid line is the Keck LWS mid-infrared spectrum of the southern nucleus, while the thick dotted line is the ISOCAM CVF spectrum (Lutz et al. 2003) scaled to match the LWS spectrum. Note that the ISOCAM spectrum contains both nuclei and the surrounding area. The thin dashed and dotted lines show the ISO SWS spectra of M82 and NGC 253 (Sturm et al. 2000), respectively, scaled to match the LWS spectrum.

beam. The two data sets show good agreement, confirming the accuracy of the photometric calibration. The LWS spectrum of the southern nucleus is overplotted, showing that the overall continuum shape (e.g., the depth of the $10 \mu \mathrm{m}$ trough) is quite similar to that of the ISOCAM spectrum, although the emission features (e.g., $11.3 \mu \mathrm{m}$ PAH) are much weaker.

\section{DISCUSSION}

\subsection{The Southern Nucleus}

As is the case with other ULIRGs (Soifer et al. 2000), the southern nucleus of NGC 6240, which dominates the luminosity output, is characterized by an extremely high surface brightness. If we take the intrinsic source area to be a circular disk with a diameter of $200 \mathrm{pc}$ on the sky, the bolometric luminosity of $7 \times 10^{11} L_{\odot}$ translates into a surface brightness of $2 \times 10^{13} L_{\odot} \mathrm{kpc}^{-2}$. This is at the high end of surface brightnesses of typical starburst galaxies (Soifer et al. 2000; Evans et al. 2003). The real surface brightness is probably even higher, since we have taken an upper limit on the size of the continuum-emitting region.

Despite this high surface brightness, the mid-infrared spectrum of the southern nucleus is similar to those of the much less luminous dusty starburst galaxies such as M82 and NGC 253. Figure 5 compares the LWS spectrum of the southern nucleus with the ISO SWS spectra of M82 and NGC 253 (Sturm et al. 2000). These spectra can be compared directly, since they both sample the light within an area of a few hundred parsecs in diameter around the nucleus. The match is reasonable in terms of the $10 \mu \mathrm{m}$ trough depth and PAH feature strengths. On the basis of the similarity of the mid-infrared spectrum to those of M82 and NGC 253, we would argue that the mid-infrared emission originates from starbursts.

The good agreement between the LWS and ISOCAM spectra seen in Figure 5 can be interpreted as a consequence of the southern nucleus dominating the total luminosity of the system. For this reason, we will not distinguish between the LWS spectrum and the ISOCAM spectrum in the following discussion once an appropriate scaling is applied to the latter to match the former.

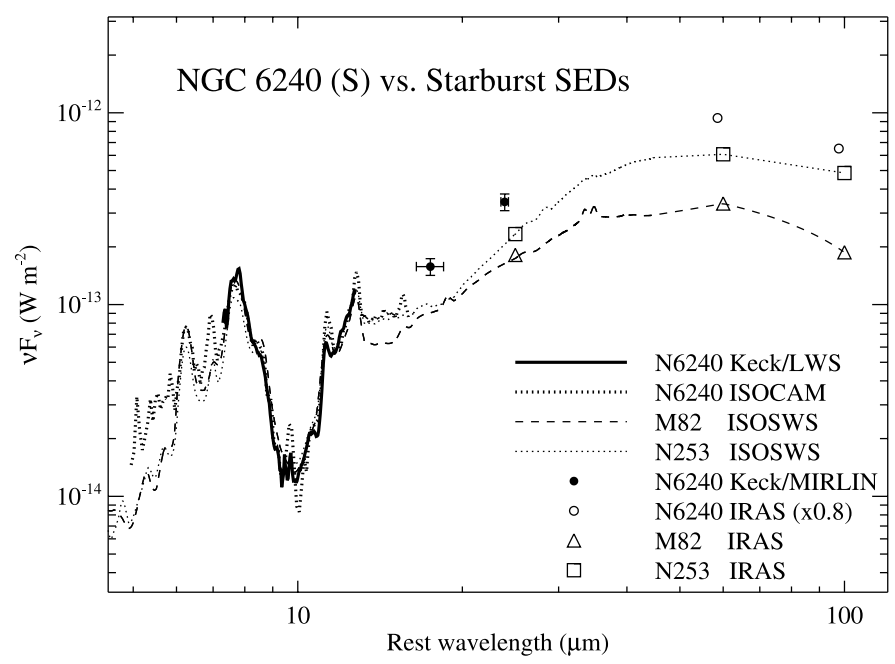

FIG. 6.-Mid-/far-infrared SEDs of the southern nucleus of NGC 6240 compared with those of the local starburst galaxies M82 and NGC 253. The ISO SWS spectra were extended to $100 \mu \mathrm{m}$ using the IRAS 25, 60, and $100 \mu \mathrm{m}$ measurements (triangles and squares) scaled such that the IRAS and ISO SWS agree at $25 \mu \mathrm{m}$. The 17.9 and $24.5 \mu \mathrm{m}$ photometric points of the NGC 6240 southern nucleus are from MIRLIN, while those at 60 and $100 \mu \mathrm{m}$ are from IRAS multiplied by a factor of 0.8 to account for the southern nucleus contribution to the total flux.

Although the 7-13 $\mu \mathrm{m}$ spectra match well, the spectrum and SED of the NGC 6240 southern nucleus outside this range are different from those of M82 and NGC 253 in three respects (Fig. 6): (1) the NGC 6240 SED rises more sharply in the midinfrared, (2) the NGC 6240 spectrum drops more slowly at $<7 \mu \mathrm{m}$, and (3) the NGC 6240 SED has more luminosity in the far-infrared. The third point is less certain because this depends on the far-infrared SED of the southern nucleus, which we cannot measure directly. In Figure 6 we estimated the southern nucleus far-infrared SED of NGC 6240 from the IRAS 60 and $100 \mu \mathrm{m}$ measurements, assuming a luminosity ratio of 4 between the two nuclei (i.e., the southern nucleus luminosity contribution is $80 \%$ ). If the true ratio is larger, as suggested by the midinfrared measurements (up to $\sim 8$ ), then the discrepancy becomes larger; on the other hand, if the true ratio is as small as the radio measurements indicate (down to $\sim 2$ ), the discrepancy, at least in comparison with NGC 253, goes away.

It is interesting that between the two SEDs of the starburst galaxies, the SED of NGC 253 is significantly closer to that of NGC 6240. NGC 253 is also known to contain an AGN, detected in the radio (Ulvestad \& Antonucci 1997) and X-ray (Weaver et al. 2002), and this might explain the similarity. In fact, as we show below, it is possible to reproduce the SED of NGC 6240 from that of M82 by adding spectral components produced by a heavily dust-obscured AGN. On the other hand, the AGN in NGC 253 is thought to be much weaker than that in NGC 6240, and therefore it may not have a significant effect on the shape of the infrared SED. In this case, the difference between the SEDs of M82 and NGC 253, although quite significant (Fig. 6), should simply be understood as a variation of properties among starburst galaxies. Although the situation is not yet clear, the simplest interpretation would be the latter; that is, there is a significant variation of properties among starburst galaxies, and therefore everything we see in the infrared spectrum and SED of NGC 6240 can still be explained by starbursts.

\subsection{The Hidden $A G N$}

Given the fact that the AGN in NGC 6240 is hidden behind a Compton-thick wall of material (Vignati et al. 1999), it is not 
surprising that there is no clear AGN signature seen in the mid-infrared. A column of Compton-thick material $\left(N_{\mathrm{H}}>2 \times\right.$ $10^{24} \mathrm{~cm}^{-2}$ ) could produce a visual extinction of $A_{V}>1000 \mathrm{mag}$, which is simply too large to be penetrated in the mid-infrared. In fact, Krabbe et al. (2001) have shown that compared with Seyfert galaxies, NGC 6240 is an order of magnitude underluminous in the mid-infrared with respect to its hard X-ray luminosity, which suggests that the AGN light is highly absorbed even in the mid-infrared.

We have estimated the maximum AGN contribution allowed by the data using a simple model. The mid-infrared radiation of the AGN was assumed to be heavily extincted so as to erase the AGN spectrum in the mid-infrared, and the absorbed midinfrared luminosity was assumed to be reemitted in the farinfrared as thermal radiation from dust. The emission resulting from this model is shown in Figure 7 and consists of the following three components: (1) starburst emission, (2) residual AGN emission after absorption, and (3) reemitted AGN emission. The M82 SED shown in Figure 6 was used to represent the starburst. A spectrum of NGC 1068 (Sturm et al. 2000) reddened by $A_{V}=70 \mathrm{mag}$ represented the residual AGN emission. The NGC 1068 spectrum was extended to longer wavelengths by extrapolating the power-law continuum at $18-45 \mu \mathrm{m}$. This extrapolation does not affect the modeling results as long as it is much less luminous than the other components in the farinfrared. The reemitted AGN was represented by a blackbody emission with a temperature of $55 \mathrm{~K}$, a value that produces a good fit to the observed SED (a similar temperature of $57 \mathrm{~K}$ was also derived by Klaas et al. [2001]). In this particular model, the luminosity of the starburst in the restricted $4.5-120 \mu \mathrm{m}$ range is $1.8 \times 10^{11} L_{\odot}$, that emitted by the AGN after extinction is $1.1 \times 10^{11} L_{\odot}$, and that of the reemitted AGN is $1.5 \times 10^{11} L_{\odot}$, which makes the total emitted luminosity ascribable to the AGN $2.6 \times 10^{11} L_{\odot}$. The intrinsic AGN luminosity could even be larger if the AGN emission is not isotropic in the infrared. This particular model assumes an intrinsic AGN luminosity of $3.3 \times 10^{11} L_{\odot}$, which was a free parameter. So, if this model prediction is correct, $\sim 20 \%$ of the AGN luminosity is not seen in our line of sight as either reemitted or residual AGN energy after absorption.

In this model, the deficiency of the M82 SED is compensated by the absorption and reemission of the AGN light. As seen in Figure 7, the AGN-related emission nicely adds luminosity to the M82 SED such that the final SED matches the observed SED. The relative contribution to the total observed luminosity is $40 \%$ from the starbursts and $60 \%$ from the AGN at $4.5-$ $120 \mu \mathrm{m}$. If we take the intrinsic AGN luminosity $\left(3.3 \times 10^{11} L_{\odot}\right)$ instead of the observed (i.e., residual plus reemitted), the AGN contribution becomes $65 \%$. Although it is not difficult to reduce the AGN contribution significantly by adopting a different starburst SED (e.g., NGC 253), this particular model indicates that it is also possible to bring up the AGN contribution level to $60 \%-65 \%$ in NGC 6240 despite the absence of any obvious AGN signatures in the mid-infrared spectrum. Given various uncertainties, these numbers are compatible with the estimates by Lutz et al. (2003) based on a number of AGN-starburst diagnostics, including the properties of the mid-infrared finestructure lines observed by the ISO SWS. The purpose of this modeling, however, is not to promote the view that the AGN dominates the luminosity output of NGC 6240, since the model presented here is just one example in which we pushed the AGN luminosity to its maximum despite some difficulties. The intention is rather to illustrate how subtle the effects can be when the SED of a heavily absorbed AGN (the residual AGN emis-

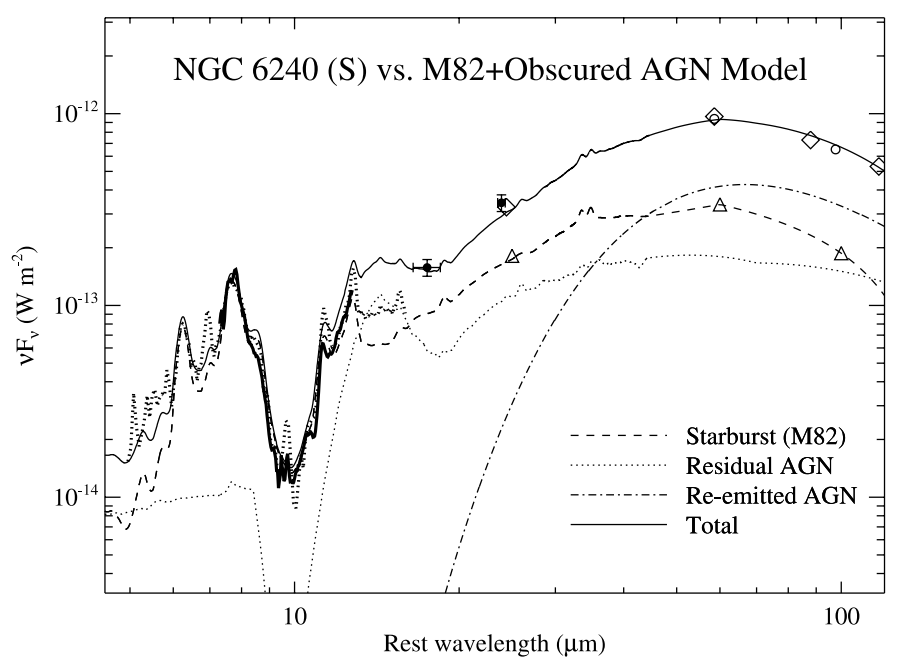

FIG. 7.-SED model fit for the southern nucleus with both starburst and AGN components. The observed mid-infrared spectra and mid-/far-infrared SED shown in Fig. 6 are reproduced by three components, as discussed in the text: (1) starburst emission (M82 spectrum; dashed line and open triangles); (2) residual AGN emission after absorption (NGC 1068 spectrum reddened by $A_{V}=70$ mag; dotted line); and (3) reemitted AGN emission (55 K blackbody radiation; dash-dotted line). The sum of these three components is shown by the solid line. The diamonds represent the measurements by Klaas et al. (2001) and were multiplied by a factor of 0.8 to simulate the fluxes from the southern nucleus. The circles represent the NGC 6240 photometric measurements shown in Fig. 6.

sion after absorption plus reemitted components) is added to that of starbursts.

Perhaps the most serious flaw of this model is the overproduction of the flux at $13-18 \mu \mathrm{m}$. It is difficult to suppress the AGN flux in this wavelength range because it corresponds to the local minimum of the extinction curve between the 10 and $18 \mu \mathrm{m}$ silicate absorption. However, given our limited knowledge of the extinction curve in this wavelength range, we do not yet consider this problem as fatal. Put in another way, if the extinction curve adopted here is correct, this is the wavelength range in which we may be able to detect heavily dust-obscured AGNs.

In fact, the choice of an extinction law affects such modeling substantially. For the residual AGN spectrum shown in Figure 7, we used the extinction law by Weingartner \& Draine (2001) for the Milky Way dust size distribution with total extinction to reddening $R_{V}=A_{V} / E(B-V)=3.1$ and increased the amount of extinction between 5 and $9 \mu \mathrm{m}$ to fit the extinction law found for the line of sight to the Galactic center (Lutz et al. 1996). This modification was necessary to suppress the AGN light and produce a good fit to the observed spectrum. Without this modification, the AGN light would have dominated the spectrum at $\lesssim 7 \mu \mathrm{m}$ with the assumed level of extinction ( $\left.A_{V}=70 \mathrm{mag}\right)$.

\subsection{The Limitations of the AGN-Starburst Power Diagnostics}

When, as seems to be the case with NGC 6240 (Krabbe et al. 2001), the AGNs are highly absorbed in the mid-infrared, the mid-infrared diagnostics (e.g., Genzel et al. 1998; Laurent et al. 2000; Imanishi \& Dudley 2000) lose their sensitivity to AGNs. The most reliable AGN-starburst power diagnostics are apparently the hard-X-ray-bolometric luminosity correlation for AGNs and the radio-infrared luminosity correlation for starbursts. However, neither of these diagnostic relations can be used to estimate the bolometric luminosity of an AGN or starbursts with an accuracy better than a factor of a few. The radio-infrared luminosity 
correlation for starbursts is quite tight over a large luminosity range, but even in the most recent and extensive compilation (Yun et al. 2001), this correlation has an intrinsic $1 \sigma$ scatter of almost a factor of $2(0.26 \mathrm{dex})$. The scatter is larger with the correlation between the AGN hard X-ray and bolometric luminosities. This means that when the starburst and AGN luminosities are comparable (i.e., within a factor of a few of each other), it is impossible to determine which dominates and by how much.

The SED variation between the two archetypal starburst galaxies M82 and NGC 253 (Fig. 6) underscores this intrinsic uncertainty associated with any AGN-starburst power diagnostics. Despite the good match of the mid-infrared spectra, their SEDs differ by a factor of a few in the far-infrared, where most of the luminosity is coming out. This would leave room for a substantial contribution from a heavily dust-obscured AGN that emits mostly in the far-infrared.

The AGN-starburst power diagnostics based on mid-infrared data would likely suffer a larger uncertainty compared with the diagnostics using hard X-ray and radio data because of the larger effects of extinction involved, which are difficult to correct accurately. As seen in Table 2, the flux ratio of the two nuclei in the mid-infrared $(7: 1)$ is significantly different from the ratios in the hard X-ray $(3: 1)$ and radio $(2: 1)$. If we take the hard X-ray and radio ratios as the luminosity ratios of the AGN and starburst components, respectively, then this means that the bolometric luminosity ratio of the two nuclei must be $2-3$, regardless of what the relative starburst/AGN luminosity contributions are. However, for some reason (most likely due to a larger extinction in the northern nucleus), this is not the case in the mid-infrared out to $24 \mu \mathrm{m}$. The far-infrared power diagnostics (e.g., the [C II] $158 \mu \mathrm{m}$ line luminosity vs. far-infrared luminosity) are more promising in principle, and according to these diagnostics, NGC 6240 is a starburst galaxy (Luhman et al. 1998, 2003). However, unless this correlation is significantly tighter than that of the radioinfrared luminosity correlation, we will again be left with the factor of a few uncertainty.

What may be even more problematic is the assumption that the infrared radiation from a ULIRG is isotropic even when it contains a dust-obscured AGN. A natural consequence of a thick dusty torus model is that most of the AGN radiation is directed perpendicular to the plane of the torus. This means that if we are looking at the torus from an edge-on direction, which may be the case for NGC 6240 considering the large extinction derived from the hard X-ray observations, most of the AGN luminosity may be invisible to us. In other words, even if the observed infrared luminosity is almost all due to starbursts, this would not necessarily exclude the existence of an equally (or even more) powerful AGN inferred from the hard X-ray observations, since the true bolometric luminosity of NGC 6240 may be substantially larger than calculated from our line of sight. Observationally, the detection of the flat (i.e., unreddened) reflected hard X-ray continuum (Iwasawa \& Comastri 1998; Boller et al. 2003) and a high-ionization [O IV] line (Genzel et al. 1998; Lutz et al. 2003) suggests that the AGN is not completely covered in all directions by Compton-thick material, which is consistent with a torus-like geometry.
For a toroidal model to work, it also requires the existence of some material that obscures the AGN in the polar directions of the torus and softens the AGN radiation. Otherwise, the various emission lines would show excitation levels higher than observed, which are those of a LINER in NGC 6240 (Heckman et al. 1983). There are indications in some Seyfert galaxies that AGN radiation is significantly softened before reaching the narrow-line regions (Alexander et al. 1999, 2000), but it remains to be seen whether such a model would work in detail to reproduce the observed properties of NGC 6240 .

\section{CONCLUSIONS}

Using the Keck telescopes, we have obtained mid-infrared images and spectra of the luminous infrared galaxy NGC 6240 with subarcsecond spatial resolutions. The main conclusions are as follows:

1. The mid-infrared (and therefore, presumably, the farinfrared) luminosity of NGC 6240 mostly originates from the two nuclei located at the positions of the southern and northern radio and hard $\mathrm{X}$-ray sources.

2. The southern nucleus is especially luminous and compact $(<200 \mathrm{pc}$ in diameter), emitting $80 \%-90 \%$ of the mid-infrared luminosity of the two nuclei.

3. The southern nucleus seems to be powered by starbursts. Its mid-infrared spectrum is similar to those of the local starburst galaxies M82 and NGC 253, and its mid-/far-infrared SED is broadly consistent with those of the starburst galaxies.

4. At the same time, because of the slight mismatch between the NGC 6240 SED and those of the starburst galaxies, a significant AGN contribution (up to $\sim 60 \%$ ) to the bolometric luminosity is also possible if the AGN is heavily obscured and emits mostly in the far-infrared.

5. Due to the intrinsic variation of properties among a given galaxy population, there is a scatter in the relationships inherent even with the most reliable and accurate AGN-starburst power diagnostics. When the starburst and AGN luminosities are within a factor of a few of each other, this makes it impossible to determine in individual galaxies whether an AGN or starbursts dominate the luminosity.

We thank E. Sturm, A. S. Evans, and H. W. W. Spoon for providing us the ISO SWS spectra, the HST NICMOS images, and the ISOCAM CVF spectrum, respectively. We would also like to thank the anonymous referee for very helpful comments. The authors wish to recognize and acknowledge the very significant cultural role and reverence that the summit of Mauna Kea has always had within the indigenous Hawaiian community. We are most fortunate to have the opportunity to conduct observations from this mountain. This research has made use of the NASA/IPAC Extragalactic Database, which is operated by the Jet Propulsion Laboratory, California Institute of Technology, under contract with the National Aeronautics and Space Administration.

\section{REFERENCES}

Alexander, T., Lutz, D., Sturm, E., Genzel, R., Sternberg, A., \& Netzer, H. 2000, ApJ, 536, 710

Alexander, T., Sturm, E., Lutz, D., Sternberg, A., Netzer, H., \& Genzel, R. 1999, ApJ, 512, 204

Armus, L., Heckman, T. M., \& Miley, G. K. 1990, ApJ, 364, 471

Beswick, R. J., Pedlar, A., Mundell, C. G., \& Gallimore, J. F. 2001, MNRAS, 325,15

Bogdanović, T., Ge, J., Max, C. E., \& Raschke, L. M. 2003, AJ, 126, 2299

Boller, Th., Keil, R., Hasinger, G., Costantini, E., Fujimoto, R., Anabuki, N., Lehmann, I., \& Gallo, L. 2003, A\&A, 411, 63

Carral, P., Turner, J. L., \& Ho, P. T. P. 1990, ApJ, 362, 434

Colbert, E. J. M., Wilson, A. S., \& Bland-Hawthorn, J. 1994, ApJ, 436, 89

Dudley, C. C. 1999 , MNRAS, 307, 553

Evans, A. S., et al. 2003, AJ, 125, 2341 
Fosbury, R. A. E., \& Wall, J. V. 1979, MNRAS, 189, 79

Fried, J. W., \& Schulz, H. 1983, A\&A, 118, 166

Gallimore, J. F., \& Beswick, R. 2004, AJ, 127, 239

Genzel, R., et al. 1998, ApJ, 498, 579

Gerssen, J., van der Marel, R. P., Axon, D., Mihos, J. C., Hernquist, L., \& Barnes, J. E. 2004, AJ, 127, 75

Heckman, T. M., Armus, L., \& Miley, G. K. 1987, AJ, 93, 276 . 1990, ApJS, 74, 833

Heckman, T. M., van Breugel, W., Miley, G. K., \& Butcher, H. R. 1983, AJ, 88, 1077

Herbst, T. M., Graham, J. R., Tsutsui, K., Beckwith, S., Matthews, K., \& Soifer, B. T. 1990, AJ, 99, 1773

Imanishi, M., \& Dudley, C. C. 2000, ApJ, 545, 701

Iwasawa, K., \& Comastri, A. 1998, MNRAS, 297, 1219

Iwasawa, K., Matt, G., Guainazzi, M., \& Fabian, A. C. 2001, MNRAS, 326, 894

Iwasawa, K., Sanders, D. B., Evans, A. S., Trentham, N., Miniutti, G., \& Spoon, H. W. W. 2005, MNRAS, 357, 565

Jones, B., \& Puetter, R. C. 1993, Proc. SPIE, 1946, 610

Joseph, R. D., \& Wright, G. S. 1985, MNRAS, 214, 87

Kii, T., Nakagawa, T., Fujimoto, R., Ogasaka, Y., Miyazaki, T., Kawabe, R., \& Terashima, Y. 1997, in X-Ray Imaging and Spectroscopy of Cosmic Hot Plasmas, ed. F. Makino \& K. Mitsuda (Tokyo: Universal Academy Press), 161

Klaas, U., et al. 2001, A\&A, 379, 823

Komossa, S., Burwitz, V., Hasinger, G., Predehl, P., Kaastra, J. S., \& Ikebe, Y. 2003, ApJ, 582, L15

Komossa, S., Schulz, H., \& Greiner, J. 1998, A\&A, 334, 110

Krabbe, A., Böker, T., \& Maiolino, R. 2001, ApJ, 557, 626

Laurent, O., Mirabel, I. F., Charmandaris, V., Gallais, P., Madden, S. C., Sauvage, M., Vigroux, L., \& Cesarsky, C. 2000, A\&A, 359, 887

Lester, D. F., Harvey, P. M., \& Carr, J. 1988, ApJ, 329, 641

Lira, P., Ward, M. J., Zezas, A., \& Murray, S. S. 2002, MNRAS, 333, 709

Luhman, M. L., Satyapal, S., Fischer, J., Wolfire, M. G., Sturm, E., Dudley, C. C., Lutz, D., \& Genzel, R. 2003, ApJ, 594, 758

Luhman, M. L., et al. 1998, ApJ, 504, L11

Lutz, D., Sturm, E., Genzel, R., Spoon, H. W. W., Moorwood, A. F. M., Netzer, H., \& Sternberg, A. 2003, A\&A, 409, 867

Lutz, D., et al. 1996, A\&A, 315, L269

Max, C. E., Canalizo, G., Macintosh, B. A., Raschke, L., Whysong, D., Antonucci, R., \& Schneider, G. 2005, ApJ, 621, 738

Mitsuda, K. 1995, Ann. NY Acad. Sci., 759, 213

Mould, J. R., et al. 2000, ApJ, 529, 786

Nakagawa, T., Kii, T., Fujimoto, R., Miyazaki, T., Inoue, H., Ogasaka, Y., \& Kawabe, R. 1999, Ap\&SS, 266, 43
Netzer, H., Lemze, D., Kaspi, S., George, I. M., Turner, T. J., Lutz, D., Boller, T., \& Chelouche, D. 2005, ApJ, 629, 739

Ressler, M. E., Werner, M. W., van Cleve, J., \& Choa, H. 1994, Exp. Astron., 3, 277

Ridgway, S. E., Wynn-Williams, C. G., \& Becklin, E. E. 1994, ApJ, 428, 609

Rieke, G. H., Cutri, R. M., Black, J. H., Kailey, W. F., McAlary, C. W., Lebofsky, M. J., \& Elston, R. 1985, ApJ, 290, 116

Rigopoulou, D., Spoon, H. W. W., Genzel, R., Lutz, D., Moorwood, A. F. M., \& Tran, Q. D. 1999, AJ, 118, 2625

Sanders, D. B., Mazzarella, J. M., Kim, D.-C., Surace, J. A., \& Soifer, B. T. 2003, AJ, 126, 1607

Sanders, D. B., \& Mirabel, I. F. 1996, ARA\&A, 34, 749

Schulz, H., Komossa, S., Berghofer, T. W., \& Boer, B. 1998, A\&A, 330, 823

Scoville, N. Z., et al. 2000, AJ, 119, 991

Shier, L. M., Rieke, M. J., \& Rieke, G. H. 1996, ApJ, 470, 222

Smith, C. H., Aitken, D. K., \& Roche, P. F. 1989, MNRAS, 241, 425

Soifer, B. T., Neugebauer, G., Matthews, K., Becklin, E. E., Ressler, M., Werner, M. W., Weinberger, A. J., \& Egami, E. 1999, ApJ, 513, 207

Soifer, B. T., Neugebauer, G., Matthews, K., Egami, E., \& Weinberger, A. J. 2002, AJ, 124, 2980

Soifer, B. T., et al. 1984, ApJ, 283, L1 . 2000, AJ, 119, 509

Solomon, P. M., Downes, D., Radford, S. J. E., \& Barrett, J. W. 1997, ApJ, 478, 144

Spoon, H. W. W., Moorwood, A. F. M., Lutz, D., Tielens, A. G. G. M., Siebenmorgen, R., \& Keane, J. V. 2004, A\&A, 414, 873

Sturm, E., Lutz, D., Tran, D., Feuchtgruber, H., Genzel, R., Kunze, D., Moorwood, A. F. M., \& Thornley, M. D. 2000, A\&A, 358, 481

Sugai, H., Malkan, M. A., Ward, M. J., Davies, R. I., \& McLean, I. S. 1997, ApJ, 481, 186

Tacconi, L. J., Genzel, R., Tecza, M., Gallimore, J. F., Downes, D., \& Scoville, N. Z. 1999, ApJ, 524, 732

Tecza, M., Genzel, R., Tacconi, L. J., Anders, S., Tacconi-Garman, L. E., \& Thatte, N. 2000, ApJ, 537, 178

Ulvestad, J. S., \& Antonucci, R. R. J. 1997, ApJ, 488, 621

van der Werf, P. P., Genzel, R., Krabbe, A., Blietz, M., Lutz, D., Drapatz, S., Ward, M. J., \& Forbes, D. A. 1993, ApJ, 405, 522

Vignati, P., et al. 1999, A\&A, 349, L57

Weaver, K. A., Heckman, T. M., Strickland, D. K., \& Dahlem, M. 2002, ApJ, 576, L19

Weingartner, J. C., \& Draine, B. T. 2001, ApJ, 548, 296

Wright, G. S., Joseph, R. D., \& Meikle, W. P. S. 1984, Nature, 309, 430

Wynn-Williams, C. G., \& Becklin, E. E. 1993, ApJ, 412, 535

Yun, M. S., Reddy, N. A., \& Condon, J. J. 2001, ApJ, 554, 803 